\title{
An integrated approach to prevent and treat respiratory failure in brain-injured patients Paolo Pelosi, Paolo Severgnini and Maurizio Chiaranda
}

\section{Purpose of review \\ Brain-injured patients are at increased risk of extracerebral organ dysfunction, in particular ventilator-associated pneumonia. The purpose of this review is to discuss functional abnormalities, clinical treatment, and possible prevention of respiratory function abnormalities in brain- injured patients.}

\section{Recent findings}

Ventilator-associated pneumonia worsens the neurologic outcome and increases the intensive care unit and hospital stay, costs, and risk of death. The respiratory dysfunction can be due to several causes, but atelectasis and/or consolidation of the lower lobes predominates in the most severe cases. Strategies should be implemented to prevent lung infections and accelerate weaning from mechanical ventilation to reduce the incidence of respiratory dysfunction and ventilator-associated pneumonia. Summary

An integrated approach including appropriate ventilatory, antibiotic, and fluid management could be extremely useful, not only to prevent and more rapidly treat respiratory failure but also to improve neurologic outcome and reduce hospital stay. Further studies are warranted to better elucidate the pathophysiology and clinical treatment of respiratory dysfunction in brain-injured patients.

\section{Keywords}

brain injury, respiratory failure, ventilator-associated pneumonia, extracerebral organ dysfunctions

Curr Opin Crit Care 11:37-42. @ 2005 Lippincott Williams \& Wilkins

Dipartimento Ambiente, Salute e Sicurezza, Universita' degli Studi dell'Insubria, Servizio di Anestesia e Rianimazione B, Ospedale di Circolo e Fondazione Macchi, Varese, Italy

Correspondence to Paolo Pelosi, Servizio di Anestesia e Rianimazione B, Ospedale di Circolo, Viale Borri 57, 21100 Varese, Italy

Tel: 00390332 278315; fax 00390332278379 ;

e-mail: ppelosi@hotmail.com, rianima.va@libero.it

Current Opinion in Critical Care 2005, 11:37-42

\section{Abbreviations}

ICU intensive care unit

VAP ventilator-associated pneumonia

\section{Introduction}

It is common knowledge that in brain-injured patients the principal morbidity and mortality is more frequently caused by the primary disease-that is, cerebral nervous system injury and its neurologic consequences [1]. Nevertheless, extracerebral organ dysfunctions are frequent in brain-injured patients, increasing the morbidity and mortality $[2,3]$. Among them, the most frequent complication is respiratory dysfunction, including pulmonary edema and pneumonia. It is now clear that an entire spectrum of pulmonary abnormalities is caused either directly or indirectly by acute brain injury. Although respiratory problems seem to play a relevant role in the clinical management of brain-injury, very few studies have investigated respiratory function abnormalities in this category of patients [4].

The causes of brain injury are traumatic, spontaneous hemorrhagic (subarachnoid or parenchymal or both), and surgical (eg, after trauma, hemorrhage, or malignancies). It is possible, however, that pulmonary-related problems and their prevention and treatment may be different between different categories of brain injury. The aim of this review is to discuss the functional abnormalities, the clinical treatment, and the possible prevention of respiratory function abnormalities in brain-injured patients.

\section{The role of extracerebral organ dysfunction in brain-injured patients}

Recently, it has been emphasized that the outcome in brain-injured patients is more frequently the result of a progressive dysfunction of organ systems remote from the site of the primary disease process-the multiple organ dysfunction process. Table 1 summarizes the average prevalence of extracerebral complications (classified into overall and severe complications) in brain-injured patients, as reported by the most recent literature. Several reports indicate that medical complications after brain injury may significantly contribute to the overall mortality rate [5]. These studies found that pulmonary alterations accounted for as many as $50 \%$ of the deaths after brain injury. The mortality was significantly higher in patients with brain injury associated with at least one organ failure than in patients with brain injury alone $(65 \%$ vs $17 \%$, respectively). Pulmonary failure was also associated with increased times in the intensive care unit (ICU) and hospital [6 $\left.6^{\bullet \bullet}\right]$.

In summary, extracerebral organ dysfunctions and in particular pulmonary failure are important causes of morbidity and mortality in brain-injured patients. 


\begin{tabular}{lcr}
$\begin{array}{l}\text { Table 1. Prevalence of extracerebral organ dysfunctions in } \\
\text { brain-injured patients }\end{array}$ & Total (\%) & Severe (\%) \\
\hline & & \\
\hline Pulmonary & 40 & 25 \\
$\quad$ Pneumonia & $<1$ & 100 \\
Embolism & 40 & 5 \\
Gut & 30 & 5 \\
Cardiac & 5 & 1 \\
$\quad$ Arrhythmia & & \\
Cardiac failure & 30 & 2 \\
Metabolic & 7 & 7 \\
$\quad$ Electrolyte disorders & 25 & 4 \\
Diabetes insipidus & 20 & 6 \\
Hepatic & 10 & 15 \\
Hematologic & & \\
Renal & &
\end{tabular}

\section{Why pulmonary complications occur in brain-injured patients}

We can identify three major causes of pulmonary complications in brain-injured patients: (1) neurogenic pulmonary edema, (2) abnormalities in ventilation-perfusion mismatch, and (3) structural parenchymal abnormalities.

\section{Neurogenic pulmonary edema}

The most dramatic pulmonary complication in brain-injured patients has been reported to be neurogenic pulmonary edema. In the $1960 \mathrm{~s}$, Simmons et al. [7] reported that $85 \%$ of their series of combat casualties from Vietnam who died with a severe isolated head injury demonstrated significant pulmonary pathologic changes, including alveolar edema, hemorrhage, and congestion, that were not the result of direct lung injury such as chest trauma; this is called neurogenic pulmonary edema. However, neurogenic pulmonary edema is very rare in civilians with brain injury, except in young patients with massive and usually rapidly fatal brain damage.

\section{Ventilation-perfusion mismatch}

Several authors have observed that most brain-injured patients with moderate to severe hypoxemia do not show evident radiographic abnormalities. Thus, it has been postulated that respiratory failure can occur without interstitial or alveolar edema, but only because of a ventilationperfusion mismatch [8].

Three main mechanisms leading to ventilation-perfusion mismatch in brain-injured patients are these:

1. redistribution in regional perfusion, which has been found to be partially mediated by the hypothalamus

2. pulmonary microembolisms, which could lead to increased dead space ventilation

3. lung surfactant depletion due to excessive sympathetic stimulation and hyperventilation

\section{Structural parenchymal abnormalities}

The main reasons for respiratory insufficiency in braininjured patients are structural parenchymal abnormalities. We can identify five main causes of structural parenchymal alterations: (1) abnormal breathing pattern, (2) release of inflammatory mediators, (3) release of catecholamines ("sympathetic storm"), (4) infectious processes, and (5) consequences of direct trauma, such as lung contusion, pneumothorax, or pain-induced hypoventilation from rib fractures.

\section{Abnormal breathing pattern}

Abnormal breathing patterns are commonly seen after brain injury. In particular, both hyperventilation and hypoventilation have been described. Hyperventilation is usually associated with periods of hypoventilation, which, together with a reduction in cough reflexes and impaired airway patency from inspissated secretions, can induce alveolar atelectasis and consolidation [9].

\section{Release of inflammatory mediators}

Brain injury causes a marked release in the brain and in the systemic circulation of inflammatory and antiinflammatory agents, which can lead to peripheral organ dysfunction, especially of the lung, and to moderate to severe immunodepression $[10,11]$. Thus, the release of these inflammatory mediators can lead to multiple organ failure, wherein the lung parenchyma seems to be a preferential and more susceptible target. However, possible further mechanisms for brain injury-related symptoms of systemic inflammation include the high incidence of aspiration pneumonia in patients who are in poor condition, which can provide a nidus for systemic inflammation. Impaired pulmonary gas exchange can further contribute to systemic inflammation, because invasive strategies of mechanical ventilation can cause volutrauma and barotrauma, which in turn can trigger the release of pulmonary cytokines.

\section{Release of catecholamines}

Brain injury is followed by prolonged sympathetic hyperactivity, which may lead to hypertension and/or tachycardia. This circulatory hyperactivity induces an increase in cerebral blood volume and/or cerebral blood flow and hence intracranial pressure. Moreover, the outcome after brain injury seems to be related to the intensity of the plasma catecholamines [12]. Catecholamines, mainly norepinephrine, have been shown to produce two particular effects on the lung: increased alveolar capillary barrier permeability and increased pulmonary lymph flow.

\section{Infectious processes}

Brain-injured patients are characterized by an increased risk for the development of ventilator-associated pneumonia (VAP) [13,14]. Its incidence is estimated to be between 
Table 2. Independent risk factors for ventilator-associated pneumonia in brain-injured patients

\begin{tabular}{lc}
\hline & Risk \\
\hline Risk factors related to brain injury & \\
Altered consciousness & 6.6 \\
Risk factors associated with the treatment of brain injury & 7.0 \\
Aspiration & 6.4 \\
Emergency intubation & 2.3 \\
Mechanical ventilation $>3$ days & \\
Risk factors associated with treatment of a general & 5.4 \\
population of critically ill patients & 5.3 \\
Reintubation & 4.8 \\
Age $>60$ y & 3.6 \\
Supine position & 2.9 \\
Previous disease & \\
Prior antibiotic use & \\
\hline
\end{tabular}

$30 \%$ and $50 \%$ of brain-injured patients, being extremely severe in only 20 to $25 \%$ of patients. Table 2 shows the independent risk factors for VAP in brain-injured patients. Altered consciousness is an important independent risk factor for VAP in most of the studies that included these patients in their research. VAP can be arbitrarily classified into early pneumonia, if it occurs within the first 4 days after admission to the ICU, and late pneumonia, if it occurs later. Early pneumonia accounts for approximately $50 \%$ of the overall cases of VAP during the ICU stay. Microorganisms can be classified into potentially pathogenic and nonpathogenic microorganisms. The most frequent causative agents of the early VAP include Staphylococcus aureus and, less frequently, Streptococcus pneumoniae and Haemophilus influen:ae. By contrast, the most frequent causative agents of late VAP are Enterobacteriaceae, Acinetobacter species, and Pseudomonas aeruginosa.

Although the predominant pathogens of early-onset pneumonia in patients with brain injury have been well established in several epidemiologic studies, the precise relation of prior upper airway, tracheobronchial, and gastric colonization patterns with the development of pneumonia and microbial patterns has not been well investigated. In conclusion, patients with brain injury are characterized by a high incidence of VAP. Patterns of colonization and pneumonia suggest that these patients can experience an alteration of airway immune defense very early during their illness. The upper airways represent the most important reservoir of subsequent tracheobronchial colonization associated with early-onset pneumonia (within 4 days). Both the upper airways and the stomach may be independent reservoirs for tracheobronchial colonization associated with late-onset pneumonia. Preventive measures to reduce the incidence of early-onset pneumonia in this population of patients may likely aim at the eradication of both upper and lower airway colonizers.

\section{Lung morphologic pattern in brain-injured patients}

The morphologic pattern of the lung parenchyma in brain-injured patients who experience respiratory insufficiency has not been fully investigated. CT of the lung in patients with VAP is characterized by marked lung densities in the dependent regions. By contrast, the nondependent regions seem to be relatively healthy and well aerated, without any ground-glass radiologic alteration. These dependent densities are poorly responsive to the application of recruitment maneuvers or high PEEP levels, but they are partially recruited when the patients are in a prone position (Fig. 1). This likely means that the main structural lung alteration during VAP in these patient is not mainly the alveolar consolidation but alterations in the peripheral airways with consequent alveolar collapse [15]. These findings can have important consequences for the prevention and treatment of VAP in these patients.

\section{Prevention of respiratory function abnormalities in brain-injured patients}

Because respiratory dysfunction plays a relevant role in the outcome of brain injury, prevention is extremely important. Unfortunately, very few studies have investigated the efficacy of specific protocols to prevent respiratory dysfunction in these patients, and most of them did not randomize the patients.

We believe that the main goals should be to prevent lung collapse and/or consolidation, to prevent lung infection, and to accelerate weaning from mechanical ventilation as soon as possible.

\section{Prevention of lung collapse and/or consolidation}

Mechanical ventilation with moderate levels of PEEP associated with recruitment maneuvers from the beginning of ventilatory treatment is likely important to avoid progressive collapse and further possible consolidation of the lung $[16,17]$. Recent studies have found that an open lung approach helps improve respiratory function in neurosurgical patients with severe respiratory failure without negative effects on cerebral physiology [18 ${ }^{\bullet}$. The addition of a preventive use of the prone position could be extremely useful to reduce worsening of the lung condition or to improve respiratory function when severe respiratory failure is established $\left[19^{\bullet}, 20\right]$.

Furthermore, the prone position has been found effective in improving tissue oxygenation of the brain in severely hypoxemic patients, with minimal negative effects of intracranial pressure and cerebral blood flow [21 $\left.{ }^{\bullet \bullet}\right]$. However, it should be remembered that both during recruitment maneuvers and the prone position, accurate monitoring of the physiologic cerebral function is warranted to minimize 
Figure 1. Lung density changes in representative brain-injured patient with ventilator-associated pneumonia
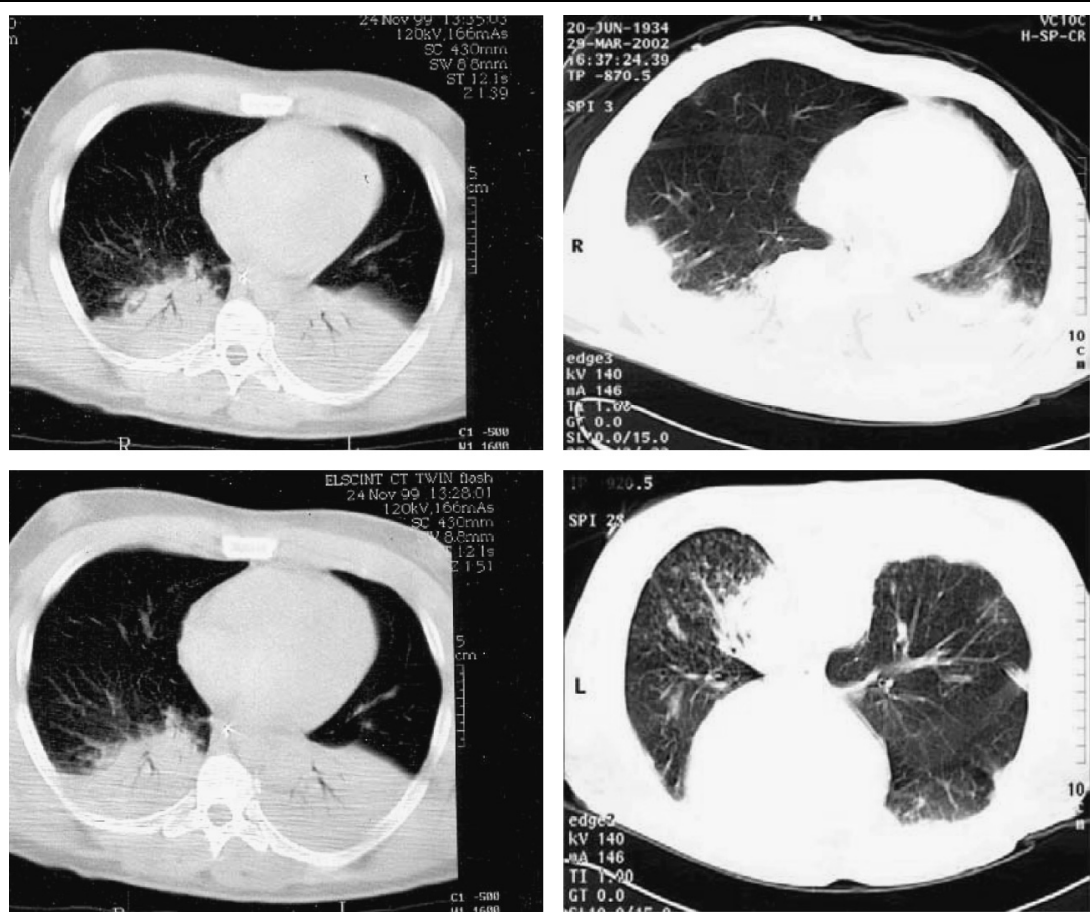

Positive end-expiratory pressure (PEEP) 5 and $15 \mathrm{~cm} \mathrm{H}_{2} \mathrm{O}$ with patient in supine position (left) and prone position (right). Lung densities were prevalently located in the dependent regions with patient in supine position, were unmodified by PEEP, but were almost completely resolved with patient in prone position.

possible negative effects on the brain induced by the maneuver itself $[22,23]$.

Careful fluid balance is likely important to reduce the progressive accumulation of fluids in the extracellular matrix, favoring the development of respiratory failure. Recent studies indicate that protocols targeted at intracranial pressure instead of cerebral perfusion can lead to a reduction in the need for fluids and consequently to a reduction of respiratory insufficiency episodes and a better neurologic outcome $[24,25]$.

Specific drugs such as low-dose corticosteroids with strict control of glucose serum levels could be useful to reduce the local and systemic inflammatory response, especially in the presence of VAP, but studies in humans are lacking $\left[26^{\bullet \bullet}\right]$. Furthermore the use of antisympathetic drugs (clonidine) and selective $\beta_{1}$ antagonists to reduce the negative systemic effects of the sympathetic storm on the lung have been found to be associated with better lung and neurologic outcome [27].

\section{Prevention of lung infection}

Several treatments have been proposed to prevent lung infection.

Antibiotic prophylaxis with second-generation cephalosporins (cefuroxime or cefoxitin) can be useful to prevent early pneumonia. However, some studies have reported an increased rate in late pneumonia if antibiotic prophylaxis is used for more than 24 hours [28].

Selective digestive decontamination has been proposed to reduce the microbiologic load in the oropharynx and in the stomach, and a few studies have shown the beneficial effects in trauma patients to reduce the incidence of VAP. However, its effects on mortality and morbidity are controversial $[29,30]$.

The upright position can also be useful to reduce VAP and the length of ICU stay in a general population of critically ill patients. The upright position is usually adopted in braininjured patients to reduce intracranial pressure when necessary [31].

Continuous oropharyngeal aspiration has been found to reduce the contamination of the upper airways and the occurrence of VAP [32].

The correct use of antacid drugs is warranted because of the increased risk of gastric hemorrhage in these patients [33].

Good nutrition improves the outcome in brain-injured patients, but the respective roles of enteral and parenteral nutrition are not clear $[34,35]$. 
Personnel hygiene protocols are warranted; in particular, a careful hand-washing policy seems to reduce the incidence of infections and improve patients' outcome [36].

\section{Accelerate weaning from intensive care unit}

Early tracheostomy may play a relevant role in weaning from the ICU and improving the clinical treatment of these patients $\left[37^{\bullet \bullet}, 38^{\bullet}\right]$. Tracheostomy is better tolerated than translaryngeal intubation, allows a better cleaning of the oropharynx, and reduces the incidence of sinusitis and the work of breathing. With the development of percutaneous techniques, the maneuver seems to be easier and safer [39]. Daily evaluation of spontaneous or minimally assisted breathing during the weaning phase is mandatory in brain-injured patients to avoid a delay in discharge from the ICU and to reduce costs. The common weaning parameters do not apply to brain-injured patients, whereas neurologic clinical evaluation plays the main role $[40,41]$.

Aggressive chest physiotherapy, positioning, and fiberoptic bronchoscopy can remove deep secretions, reducing the risk for the development of VAP, although clinical data are scanty.

Finally, daily interruption of continuous sedation decreased the length of time of ventilator use in a general population of critically ill patients [42]. However, in brain-injured patients, the optimal level of sedation is controversial because although sedation is necessary to limit intracranial hypertension crisis, it can delay the start of weaning and prolong the duration of mechanical ventilation.

\section{Conclusion}

In conclusion, brain-injured patients are at an increased risk of extracerebral organ dysfunctions, in particular VAP, leading to a worse neurologic outcome. In these patients, VAP is mainly characterized by atelectasis or consolidation of the lower lobes. Thus, strategies should be implemented to prevent lung collapse and/or consolidation and lung infections and to accelerate weaning from mechanical ventilation. We believe that an integrated approach could be extremely useful, not only to prevent or more rapidly treat respiratory failure but also to improve neurologic outcome and reduce time in the ICU.

\section{Acknowledgments}

The authors thank the medical and nursing staff of the Intensive Care Unit (Servizio Anestesia e Rianimazione B) of the Ospedale di CircoloVarese for their valuable contributions.

\section{References and recommended reading}

Papers of particular interest, published within the annual period of review, have been highlighted as:

- of special interest

-. of outstanding interest

1 Kassell NF, Torner JC, Haley EC, et al. The international cooperative study on the timing of aneurysm surgery: Part 1 and Part 2. Overall management results. J Neurosurg 1990; 73:18-47.
2 Solenski N, Haley C, Kassell NF, et al. Medical complications of aneurysmal subarachnoid hemorrhage: a report of the multicenter, cooperative aneurysm study. Crit Care Med 1995; 23:1007-1017.

3 Clifton GC, Miller ER, Choi SC, et al. Lack of effect of induction of hypothermia after acute brain injury. N Engl J Med 2000; 344:556-563.

4 Demling R, Riessen R: Pulmonary dysfunction after cerebral injury. Crit Care Med 1990; 18:768-774.

5 Gruber A, Reinprecht A, Illievich UM, et al. Extracerebral organ dysfunction and neurologic outcome after aneurysmal subarachnoid hemorrhage. Crit Care Med 1999; 27:505-514.

6 Holland MC, Mackersie RC, Morabito D, et al. The development of acute lung

-. injury is associated with worse neurologic outcome in patients with severe traumatic brain injury. J Trauma 2003; 55:106-111.

This paper describes the strict association between the development of acute lung injury and worse neurologic outcome in a large series of patients with head injury.

7 Simmons RL, Martin AM, Heisterkamp CA, et al. Respiratory insufficiency in combat casualties: pulmonary edema following head injury. Ann Surg 1969; 170:39-44.

8 Schumaker $\mathrm{P}$, Rhodes $\mathrm{G}$, Newell J, et al. Ventilation perfusion imbalance after head injury. Am Rev Respir Dis 1979; 119:33-43.

9 North J, Jennett S: Abnormal breathing patterns associated with acute brain damage. Arch Neurol 1974; 31:338-344.

10 Holmin S, Schalling M, Hojeberg B, et al. Delayed cytokines expression in rat brain following experimental contusion. J Neurosurg 1997; 86:493-504.

11 Mathiesen T, Anderson B, Loftenius A, et al. Increased interleukin-6 levels in cerebrospinal fluid following subarachnoid hemorrhage. J Neurosurg 1993 78:562-567.

12 Woolf PD, Hamill RW, Lee LA, et al. The predictive value of catecholamines in assessing outcome in traumatic brain injury. J Neurosurg 1987; 66:875882.

13 Sirvent JM, Torres A, Vidaur L, et al. Tracheal colonisation within 24 hours of intubation in patients with head trauma: risk factor for developing early onset ventilator associated pneumonia. Intensive Care Med 2000; 26:13691372.

14 Ewig S, Torres A, El-Ebiary M, et al. Bacterial colonization pattern in mechan ically ventilated patients with traumatic and medical head injury: incidence, risk factors, and association with ventilator associated pneumonia. Am J Respir Crit Care Med 1999; 159:188-198.

15 Pelosi P, Brazzi L, Gattinoni L: Prone position in acute respiratory distress syndrome. Eur Respir J 2002; 20:1017-1028.

16 Cooper KR, Boswell PA, Choi SC: Safe use of PEEP in patients with severe head injury. J Neurosurg 1985; 63:552-555.

17 Miller RS, Nelson LD, Di Russo SM, et al. High level positive end-expiratory pressure management in trauma-associated adult respiratory distress syndrome. J Trauma 1992; 33:284-290.

18 Wolf S, Shurer L, Trost HA, et al. The safety of open lung approach in neu- rosurgical patients. Acta Neurochir 2002; 81(Suppl):99-101.

This is one of a few studies to investigate the role of the open lung approach in neurosurgical patients with severe respiratory failure.

19 Beuret $\mathrm{P}$, Carton MJ, Nourdine K, et al. Prone position as prevention of lung - injury in comatose patients: a prospective, randomised, controlled trial. Intensive Care Med 2004; 28:564-569.

This is the first prospective randomized trial to show that the prone position can be useful to prevent worsening of lung conditions in brain-injured patients.

20 Voggenreiter G, Neudeck F, Aufmkolk M, et al. Intermittent prone positioning in the treatment of severe and moderate post traumatic lung injury. Crit Care Med 1999; 27:2375-2382.

21 Reinprecht $A$, Greber $H$, Wolfsberger $S$, et al. Prone position in subarachnoid

- hemorrhage patients with acute respiratory distress syndrome: effects on cerebral tissue oxygenation and ICP. Crit Care Med 2003; 31:18311838.

In this study the authors showed that the prone position improves not only systemic but also brain tissue oxygenation, with minimal effect on intracranial pressure, in a selected population of patients with subarachnoid hemorrhage and severe respiratory failure.

22 Bein T, Kuhr LP, Bele S, et al. Lung recruitment manoeuvre in patients with cerebral injury: effects on intracranial pressure and cerebral metabolism. Intensive Care Med 2002; 28:554-558.

23 Diringer MN: Subarachnoid haemorrhage: a multiple organ system disease. Crit Care Med 2003; 31:1884-1885.

This nice editorial discusses the possible beneficial and negative aspects of the prone position in brain-injured patients. 
JOBNAME: cocc 11\#1 2005 PAGE: 6 OUTPUT: Wed December 29 19:17:17 2004

lww/cocc/91165/COCC11105

\section{Respiratory system}

24 York J, Arrillaga A, Graham R, et al. Fluid resuscitation of patients with multiple injuries and severe closed head injury: experience with an aggressive fluid resuscitation strategy. J Trauma 2000; 48:376-380.

25 Contant $C F$, Valadka $A B$, Gopinath SP, et al. Adult respiratory distress syndrome: a complication of induced hypertension after severe head injury J Neurosurg 2001; 95:560-568.

26 Rocco PR, Souza AB, Faffe DS, et al. Effect of corticosteroid on lung paren- $\quad$ chyma remodelling at an early phase of acute lung injury. Am J Respir Crit Care Med 2003; 168:677-684.

In this experimental study, the authors show the beneficial effects of low-dose corticosteroids on the lung parenchyma in primary but not in secondary acute respiratory distress syndrome.

27 Asgeirsson $\mathrm{B}$, Grande $\mathrm{PO}$, Nordstrom $\mathrm{CH}$, et al. Effects of hypotensive treatment with $\alpha_{2}$ agonist and $\beta_{1}$ antagonist on cerebral hemodynamic in severely injured patients. Acta Anaesthesiol Scand 1995; 39:347-351.

28 Sirvent JM, Torres A, El-Ebiabry M, et al. Protective effect of intravenously administered cefuroxime again nosocomial pneumonia in patients with structural coma. Am J Respir Crit Care Med 1997; 155:1729-1734.

29 Korinek AM, Laisne MJ, Nicolas MH, et al. Selective decontamination of the digestive tract in neurosurgical intensive care patients: a double blind, randomised placebo controlled trial. Crit Care Med 1993; 21:14661473.

30 Leone M, Bougoin A, Giuly E, et al. Influence on outcome of ventilator associated pneumonia in multiple trauma patients with head trauma treated with selected digestive decontamination. Crit Care Med 2002; 30:17411746.

31 Drakulovic MB, Torres A, Bauer TT, et al. Supine body position as a risk factor for nosocomial pneumonia in mechanically ventilated patients: a randomized trial. Lancet 1999; 354:1851-1858.

32 Shorr AF, O'Malley PG: Continuous subglottic suctioning for the prevention of ventilator associated pneumonia: potential economic implications. Chest 2001; 119:228-235.
33 Kamada T, Fusamoto $\mathrm{H}$, Kawano $\mathrm{S}$, et al. Gastrointestinal bleeding following head injury: a clinical study of 433 cases. J Trauma 1977; 17:44-47.

34 Taylor SJ, Fettes SB, Jewkes C, et al. Prospective, randomized, controlled trial to determine the effect of early enhanced enteral nutrition clinical outcome in mechanically ventilated patients suffering head injury. Crit Care Med 1999; 27:2525-2531.

35 Rapp RP, Young B, Twyman D, et al. The favourable effect of early parentera feeding on survival in head injured patients. J Neurosurg 1983; 58:906-912.

36 Bischoff WE, Reynolds TM, Sessler CN, et al. Hand-washing compliance by health care workers: the impact of introducing an accessible, alcohol-based hand antiseptic. Arch Intern Med 2000; 160:1017-1021.

37 Rumbak MJ, Newton M, Truncale T, et al. A prospective randomised study - comparing early percutaneous dilational tracheostomy to prolonged translaryngeal intubation (delayed tracheotomy) in critically ill medical patients. Crit Care Med 2004; 32:1689-1694.

This nice prospective randomized trial shows that early percutaneous dilationa tracheostomy reduces weaning time compared with delayed tracheostomy in a large population of medical critically ill patients.

38 Arabi Y, Haddad S, Shirawi N, et al. Early tracheostomy timing and duration of - weaning in patients with respiratory failure. Crit Care 2004; 8:R347-R352. This observational study in trauma patients reports reduced weaning time by the use of early tracheostomy compared with prolonged translaryngeal intubation.

39 Pelosi P, Severgnini P: Tracheostomy must be individualized! Crit Care 2004 8:322-324.

40 Coplin WM, Pierson DJ, Cooley KD, et al. Implications of extubation delay in brain injured patients meeting standard weaning criteria. Am J Respir Crit Care Med 2000; 161:1530-1536.

41 Namen AH, Ely EW, Tatter SB, et al. Predictors of successful extubation in neurosurgical patients. Am J Respir Crit Care Med 2001; 163:658-664.

42 Kress JP, Pohlman AS, O'Connor MF, et al. Daily interruption of sedative infusions in critically ill patients undergoing mechanical ventilation. $\mathrm{N}$ Eng J Med 2000; 342:1471-1477. 